\title{
Ponatinib-Induced Graft-versus-Host Disease/ Graft-versus-Leukemia Effect in a Patient with Philadelphia-Positive Acute Lymphoblastic Leukemia without the T315I Mutation Relapsing after Allogeneic Transplant
}

\author{
Annamaria Petrungaro ${ }^{a} \quad$ Massimo Gentile $^{a} \quad$ Carla Mazzone $^{a}$ Rosa Greco ${ }^{a}$ \\ Giuseppina Uccello ${ }^{a}$ Anna Grazia Recchiac ${ }^{c}$ Laura De Stefanoc \\ Sabrina Bossio ${ }^{c}$ Angela Palummo $^{c}$ Rosellina Morellib ${ }^{b}$ Caterina Musolino $^{d}$ \\ Fortunato Morabito ${ }^{a, c}$ Ernesto Vigna ${ }^{a}$ \\ ${ }^{a}$ Hematology Unit and ${ }^{b}$ Nuclear Medicine Unit, Department of Onco-Hematology, AO of Cosenza, and \\ 'Biotechnology Research Unit, Aprigliano, AO/ASP of Cosenza, Cosenza, and d Division of Hematology, \\ University of Messina, Messina, Italy
}

\section{Established Facts}

- Ponatinib is a highly effective treatment for Philadelphia-positive acute lymphoblastic leukemia with and without T315I mutation, and it is well tolerated.

\section{Novel Insights}

- Ponatinib as a single-agent treatment can induce molecular complete responses in Philadelphia-positive acute lymphoblastic leukemia patients without T315I mutation after early relapse following allogeneic stem cell transplantation, and it can induce a graft-versus-host disease/graft-versus-leukemia (GVHD/GVL) effect through immunomodulatory activity.

\section{Keywords}

Ponatinib · Acute lymphoblastic leukemia •

Philadelphia-positive chromosome

\section{Abstract}

We describe the case of a patient with Philadelphia-positive acute lymphoblastic leukemia treated with dasatinib plus steroids as first-line therapy, who achieved a major molecu-

\section{KARGER}

(C) 2017 S. Karger AG, Basel

E-Mail karger@karger.com

www.karger.com/che lar response (MMR) before undergoing matched, unrelated donor allogeneic stem cell transplant. Eleven months after the transplant, she experienced molecular relapse. Mutational screening showed negativity for the T315I mutation, The patient underwent a salvage chemotherapy regimen with clofarabine + cyclophosphamide + steroids and ponatinib (clofarabine $70 \mathrm{mg}$ i.v., days 1-5, cyclophosphamide $700 \mathrm{mg}$ i.v., days $1-5$, and ponatinib $45 \mathrm{mg}$ p.o., daily starting at day 15). We observed a rapid decrease in minimal residual

Annamaria Petrungaro

UOC Ematologia, Azienda Ospedaliera di Cosenza Viale della Repubblica snc IT-87100 Cosenza (Italy)

E-Mail annamaria.petrungaro@gmail.com 
disease on molecular assessment with an MMR of P190-BCR$A B L / A B L=0.01 \%$ confirmed by bone marrow revaluations at days $+23,+59,+108$, and +191 after the first day of salvage chemotherapy. After starting ponatinib, the patient experienced skin graft-versus-host disease, suggesting that the efficacy of ponatinib could be related not only to the direct antileukemic effect but also to its ability to promote an indirect graft-versus-leukemia effect. Ponatinib treatment was well tolerated and considered safe with easily manageable side effects.

(c) 2017 S. Karger AG, Basel

\section{Introduction}

The Philadelphia chromosome $(\mathrm{Ph})$, resulting from a balanced translocation between chromosome 9 and 22, is the most frequent cytogenetic abnormality in leukemia and represents the cytogenetic alteration most often associated with acute lymphoblastic leukemia (ALL). The formation of the BCR/ABL fusion gene derived from the $t(9 ; 22)$ translocation occurs in almost $20-30 \%$ of adults with ALL and in almost 3\% of pediatric patients. The frequency of the Ph chromosome in ALL increases with age and is present in over $50 \%$ of patients over 50 years of age [1].

At the time of diagnosis, when compared with Ph-negative ALL, $\mathrm{Ph}$-positive $(\mathrm{Ph}+\mathrm{ALL})$ patients generally present with a higher number of white blood cells. At the onset of the disease, the central nervous system is involved in approximately $5 \%$ of cases, but the possibility of central nervous system involvement is present in all stages of the disease.

Before the era of tyrosine kinase inhibitors (TKIs) the prognosis of $\mathrm{Ph}+\mathrm{ALL}$ patients was traditionally poor; the percentage of complete remissions obtained by conventional or intensive treatment regimens ranged from 60 to $70 \%$ and the percentage of long-term survival was 35$40 \%$ in children to less than $20 \%$ in adults [2].

Imatinib, dasatinib, and ponatinib are TKIs directed against the ABL tyrosine kinase and have been approved for the treatment of Ph+ ALL. These agents have radically changed the clinical course of ALL in these patients [3-6]. TKIs can be used as monotherapy or in combination with chemotherapy and result in hematological remission rates exceeding 90\% [7].

Although outcome has improved substantially with TKI-based regimens compared with historic controls, allogeneic stem cell transplantation in first remission provides the best chance of cure for the majority of eligible patients. However, relapse due to the development of resistance mutations in the ABL kinase domain remains a major obstacle $[4,5]$.

In fact, in $50 \%$ of cases relapses are due to the appearance of molecular mutations, such as the T315I mutation associated with a TKI-resistant BCR/ABL1 clone, and therefore are not responsive to the first- and secondgeneration TKIs imatinib and dasatinib, and nilotinib $[4,5]$.

Ponatinib (AP24534) is a potent oral TKI that blocks native and mutated $\mathrm{BCR} / \mathrm{ABL}$ [7]. In vitro experiments have shown that ponatinib has potent activity against native $\mathrm{BCR} / \mathrm{ABL}$ protein and against all tested mutant forms of BCR/ABL, including the gatekeeper mutant T315I [7]. Three clinical trials have confirmed the efficacy of ponatinib in the relapsed and front-line setting in $\mathrm{Ph}+\mathrm{ALL}$ patients, even in the presence of the T315I mutation. These data support the characterization of ponatinib as a pan-BCR/ABL inhibitor and therefore supports its use in $\mathrm{Ph}+\mathrm{ALL}$ patients resistant or intolerant to dasatinib and for whom subsequent treatment with imatinib is not clinically appropriate, or in patients harboring the T315I mutation [8-10].

\section{Case Report}

We describe the case of a 43-year-old Bulgarian female patient, who had previously undergone splenectomy for spontaneous rupture of the spleen. In September 2014, the patient presented to the emergency room complaining of chest pain, asthenia, and anxiety. Routine blood count showed lymphocytosis (white blood cell count: $96,000 / \mathrm{mm}^{3}$ ), and the patient was admitted to our department for further investigation and treatment.

During hospitalization she was diagnosed with $\mathrm{Ph}+\mathrm{ALL}$ with a quantitative assessment of the BCR/ABL (P190 e1a2) molecular transcript equal to $87.008 \%$ at onset.

Thus, daily dasatinib $140 \mathrm{mg}$ plus steroids were started as firstline "chemo-free" therapy. We performed a single lumbar puncture at diagnosis and for prophylaxis purposes. The liquor test results were negative. She was treated with methotrexate $(12 \mathrm{mg})$, cytarabine (40 mg), and methylprednisolone (40 mg).

Due to the absence of an available HLA-identical bone marrow donor, a search for a matched unrelated donor was initiated in order to begin consolidation therapy with allogeneic hematopoietic stem cells (HSC).

In May 2015, the patient underwent a matched unrelated donor allogeneic transplant after conditioning chemotherapy regimen with busulfan + cyclophosphamide + Thymoglobulin-ATG (busulfan i.v. $3.2 \mathrm{mg} / \mathrm{kg} /$ day, days -7 to -4 , cyclophosphamide $60 \mathrm{mg} /$ $\mathrm{kg} /$ day, days -3 and -2 , ATG $2.5 \mathrm{mg} / \mathrm{kg} /$ day, days -3 and -2 ).

A major molecular response (MMR) (P190 BCR/ABL transcript equal to $0.002 \%$ ) following the matched unrelated donor transplant was achieved at day 134 , while a hematological response and complete chimerism (100\%) were achieved on day 187 ; on the
354

Chemotherapy 2017;62:353-356

DOI: $10.1159 / 000477714$
Petrungaro et al. 
Fig. 1. Bone marrow transcript levels of BCR/ABL1. PDN, prednisone; MUD, matched unrelated donor transplantation; Clo, clofarabine; CTX, cyclophosphamide.

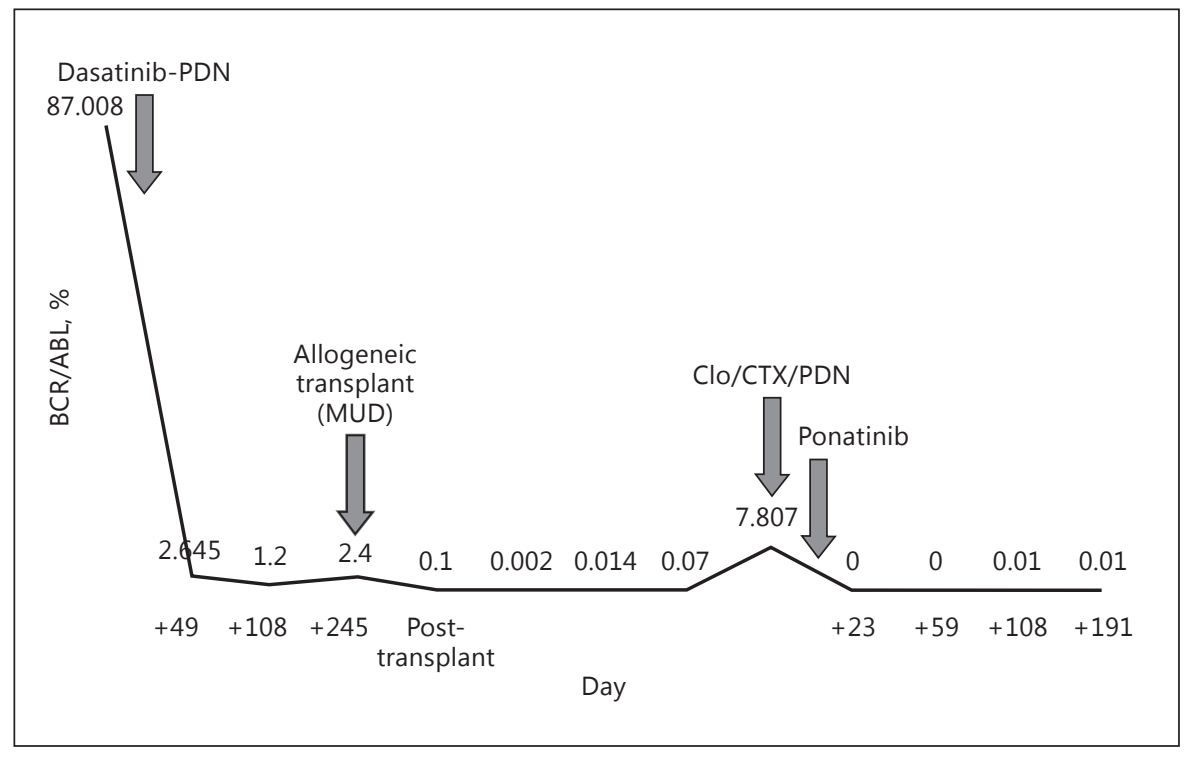

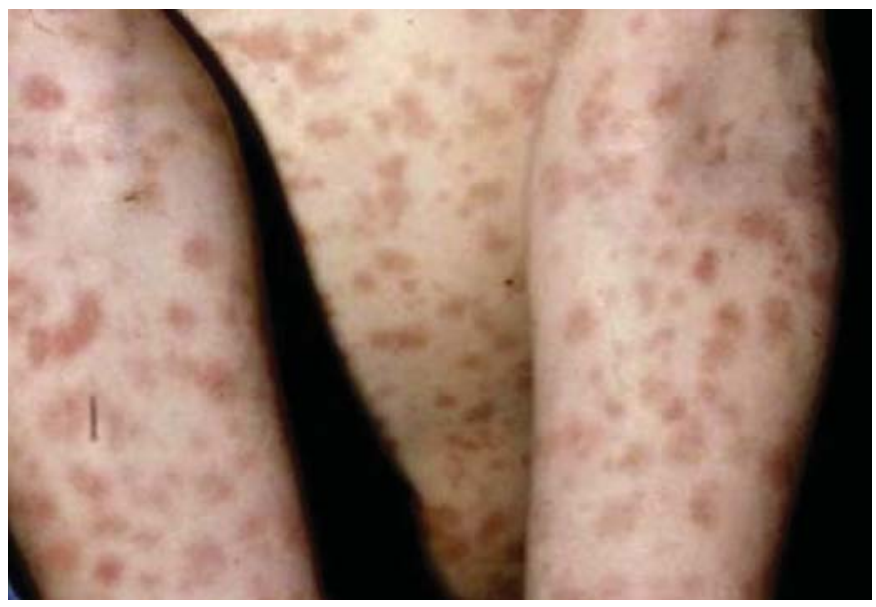

Fig. 2. Cutaneous rash.

same day, immunosuppressive therapy (cyclosporine) was discontinued. Two subsequent evaluations of P190 BCR/ABL at days 210 and 239 confirmed an MMR.

The posttransplant period was complicated by a fungal and bacterial lung infection with sputum positive for Candida albicans and Staphylococcus haemoliticus and by peripheral neuropathy (WHO grade 2).

Eleven months after the HSC transplant, at day 267, the patient experienced an increase in detectable P190 BCR/ABL transcript levels $(0.07 \%)$ without any evidence of T315I mutation. In March 2016 a new molecular evaluation showed a further rise of the P190 $\mathrm{BCR} / \mathrm{ABL}$ transcript (7\%), thus the patient was admitted to our ward and underwent a salvage chemotherapy regimen with clofarabine + cyclophosphamide + steroids and ponatinib (clofarabine $70 \mathrm{mg}$, days $1-5$, cyclophosphamide $700 \mathrm{mg}$, days $1-5$, and

Ponatinib in a Patient with $\mathrm{Ph}+\mathrm{ALL}$ ponatinib $45 \mathrm{mg}$, daily starting on day 15). We observed a rapid decrease of minimal residual disease at molecular assessment with an MMR of P 190 of $0.01 \%$ confirmed by bone marrow revaluations on days $+23,+59,+108$, and +191 following the first day of salvage chemotherapy (Fig. 1).

After starting ponatinib, on day 60 we observed persistent lymphocytosis and the patient experienced a skin GVHD-like reaction with nettle rash (Fig. 2). Immunophenotyping analysis from peripheral blood showed an increase of T-suppressor lymphocytes $(\mathrm{CD} 8+=2,422$ cells $/ \mu \mathrm{L})$ and natural killer T cells $(3,550$ cells $/ \mu \mathrm{L})$ associated with a reduction of CD $4+\mathrm{T}$ cells $(278$ cells $/ \mu \mathrm{L})$ which resolved about 2 weeks after oral steroid therapy (prednisone 1 $\mathrm{mg} / \mathrm{kg}$ for 15 days).

To date, this patient continues to show hematologic remission and MMR. She is continuing therapy with ponatinib $45 \mathrm{mg}$ /day with reasonable tolerance in the absence of hematological, cardiovascular, infectious, or endocrine complications.

\section{Discussion}

Here, we describe the case of a Ph+ ALL patient without T315I mutation, with early relapse after HSC transplant, who achieved a persistent complete response with negative minimal residual disease after treatment with single-agent ponatinib. Detectable levels of $\mathrm{BCR} / \mathrm{ABL}$ transcripts are predictive of a relapse of the disease [11], even though 2 months after allogeneic transplant there was no evidence of a hematological relapse (bone marrow morphology indicated $<5 \%$ blasts). Considering the rapid rise in transcript levels during longitudinal monitoring, with an increase of approximately 2 logs in transcript levels, chemotherapy was initiated. 
We would like to highlight the surprisingly fast, deep, and persistent molecular response obtained by ponatinib in a patient with a very poor prognosis. Secondly, TKI therapy with single-agent ponatinib after transplantation is feasible and may reduce the incidence of relapse and improve outcome after allogeneic HSC transplant in patients with high-risk $\mathrm{Ph}+$ leukemia.

Furthermore, this case suggests that the efficacy of ponatinib could be related not only to the direct antileukemic effect but also to its ability to promote an indirect GVL effect. There is no data currently available on the immunomodulatory effects of ponatinib, but it is possible to hypothesize a potential immunological role for this TKI, similar to that observed in previous studies for imatinib, dasatinib, and nilotinib [12]. However, the molecular mechanism involved in this response remains unclear, and there are no specific data available in this regard in the medical literature. Furthermore, in our case, as also observed for the other available TKIs, we observed an increase in the number of circulating CD8+ and natural killer $\mathrm{T}$ cells and a reduction in the number of $\mathrm{CD} 4+\mathrm{T}$ cells, which may be correlated with the onset of GVHD and GVL. Recently, a similar case report described a patient with Ph+ ALL with the acquisition of T315I mutation who was treated with ponatinib at relapse after an allogeneic transplant. This patient also experienced a skin GVHD, suggesting that the efficacy of ponatinib could be related to its ability to promote an indirect GVL effect [10]. This finding reinforces the use of immunotherapy in the treatment of onco-hematological diseases [13]. Finally, ponatinib treatment is considered safe with easily manageable side effects, although a careful evaluation of adverse events is required.

In conclusion, our case suggests that ponatinib may be highly effective and safe as a monotherapy in Ph+ ALL patients without the T315I mutation who relapse following allogeneic stem cell transplantation, both for its significant antileukemic actions and for its ability to induce a GVHD-like/GVL effect.

\section{Disclosure Statement}

The authors declare no financial or other potential conflicts of interest.

\section{References}

1 Mancini M, Scappaticci D, Cimino G, et al: A comprehensive genetic classification of adult acute lymphoblastic leukemia (ALL): analysis of the GIMEMA 0496 protocol. Blood 2005; 105:3434-3441.

2 Moorman AV, Harrison CJ, Buck GA, et al: Karyotype is an independent prognostic factor in adult acute lymphoblastic leukemia (ALL): analysis of cytogenetic data from patients treated on the Medical Research Council (MRC) UKALLXII/Eastern Cooperative Oncology Group (ECOG) 2993 trial. Blood 2007;109:3189-3197.

3 Sanford DS, Kantarjian H, O'Brien S, et al: The role of ponatinib in Philadelphia chromosome-positive acute lymphoblastic leukemia. Expert Rev Anticancer Ther 2015;15: 365-373.
4 Malagola M, Papayannidis C, Baccarani M: Tyrosine kinase inhibitors in $\mathrm{Ph}+$ acute lymphoblastic leukaemia: facts and perspectives. Ann Hematol 2016;95:681-693.

5 Liu-Dumlao T, Kantarjian H, Thomas DA, O'Brien S, et al: Philadelphia-positive acute lymphoblastic leukemia: current treatment options. Curr Oncol Rep 2012;14:387-394.

6 Kosior K, Lewandowska-Grygiel M, Giannopoulos K: Tyrosine kinase inhibitors in hematological malignancies. Postepy Hig Med Dosw 2011;65:819-828.

7 Thomas X: Chemotherapy plus ponatinib: a new standard for Ph-positive ALL? Lancet Oncol 2015;16:1451-1453.

8 Hirschbuehl K, Rank A, Pfeiffer T, et al: Ponatinib given for advanced leukemia relapse after allo-SCT. Bone Marrow Transplant 2015; 50:599-600.

9 Maino E, Sancetta R, Viero P, et al: Current and future management of $\mathrm{Ph} / \mathrm{BCR}-\mathrm{ABL}$ positive ALL. Expert Rev Anticancer Ther 2014; 14:723-740.
10 Renzi D, Marchesi F, De Angelis G, et al: Ponatinib induces a persistent molecular response and graft-versus-host disease/graftversus-leukemia effect in a patient with Philadelphia-positive acute lymphoblastic leukemia with a T315I mutation following early relapse after allogeneic transplant. Chemotherapy 2016;62:58-61.

11 Monika B, et al: Has MRD monitoring superseded other prognostic factors in adult ALL? Blood 2012;120:4470-4448.

12 Kreutzman A, Porkka K, Mustjoki S: Immunomodulatory effects of tyrosine kinase inhibitors. Int Trends Immun 2013:1:17-28.

13 Alatrash G, Daver N, Mittendorf EA, et al: Targeting immune checkpoints in hematologic malignancies. Pharmacol Rev 2016;68: 1014-1025. 